MATHEMATICS OF COMPUTATION

Volume 70, Number 235, Pages 1155-1167

S 0025-5718(00)01253-9

Article electronically published on March 28, 2000

\title{
A THREE-PARAMETER FAMILY OF NONLINEAR CONJUGATE GRADIENT METHODS
}

\author{
Y. H. DAI AND Y. YUAN
}

\begin{abstract}
In this paper, we propose a three-parameter family of conjugate gradient methods for unconstrained optimization. The three-parameter family of methods not only includes the already existing six practical nonlinear conjugate gradient methods, but subsumes some other families of nonlinear conjugate gradient methods as its subfamilies. With Powell's restart criterion, the three-parameter family of methods with the strong Wolfe line search is shown to ensure the descent property of each search direction. Some general convergence results are also established for the three-parameter family of methods. This paper can also be regarded as a brief review on nonlinear conjugate gradient methods.
\end{abstract}

\section{INTRODUCTION}

Consider the unconstrained optimization problem

$$
\min _{x \in R^{n}} f(x),
$$

where $f$ is a smooth function and its gradient is available. Conjugate gradient methods are a class of important methods for solving (1.1), especially for large scale problems, which have the following form:

$$
\begin{aligned}
x_{k+1} & =x_{k}+\alpha_{k} d_{k}, \\
d_{k} & = \begin{cases}-g_{k}, & \text { for } k=1, \\
-g_{k}+\beta_{k} d_{k-1}, & \text { for } k \geq 2,\end{cases}
\end{aligned}
$$

where $g_{k}=\nabla f\left(x_{k}\right), \alpha_{k}$ is a stepsize obtained by a one-dimensional line search and $\beta_{k}$ is a scalar. The strong Wolfe conditions, namely,

$$
\begin{aligned}
& f\left(x_{k}+\alpha_{k} d_{k}\right)-f_{k} \leq \delta \alpha_{k} g_{k}^{T} d_{k}, \\
& \left|g\left(x_{k}+\alpha_{k} d_{k}\right)^{T} d_{k}\right| \leq-\sigma g_{k}^{T} d_{k},
\end{aligned}
$$

where $0<\delta<\sigma<1$, are often imposed on the line search (in this case, we call the line search the strong Wolfe line search). The scalar $\beta_{k}$ is chosen so that the method (1.2) - (1.3) reduces to the linear conjugate gradient method in the case when $f$ is convex quadratic and exact line search $\left(g\left(x_{k}+\alpha_{k} d_{k}\right)^{T} d_{k}=0\right)$ is used.

Received by the editor January 22, 1999 and, in revised form, September 7, 1999.

2000 Mathematics Subject Classification. Primary 49M37, 65K05, 90C30.

Key words and phrases. Unconstrained optimization, conjugate gradient methods, line search, global convergence.

Research partly supported by Chinese NSF grants 19525101, 19731010 and 19801033.

(C)2000 American Mathematical Society 
For general functions, however, different formulae for scalar $\beta_{k}$ result in distinct nonlinear conjugate gradient methods. Several famous formulae for $\beta_{k}$ are the Fletcher-Reeves (FR), Polak-Ribière-Polyak (PRP), and Hestenes-Stiefel (HS) formulae (see $[15 ; 26,27 ; 18]$ ), which are given by

$$
\begin{aligned}
& \beta_{k}^{F R}=\frac{\left\|g_{k}\right\|^{2}}{\left\|g_{k-1}\right\|^{2}}, \\
& \beta_{k}^{P R P}=\frac{g_{k}^{T} y_{k-1}}{\left\|g_{k-1}\right\|^{2}}, \\
& \beta_{k}^{H S}=\frac{g_{k}^{T} y_{k-1}}{d_{k-1}^{T} y_{k-1}},
\end{aligned}
$$

respectively, where $\|\cdot\|$ means the Euclidean norm and $y_{k-1}=g_{k}-g_{k-1}$. The convergence properties of the FR, PRP, and HS methods have been studied in many references, for example [1, [5, 7, 16, 17, 21, 25, 28, 31. However, if the condition imposed on $\sigma$ in (1.5) is only that $\sigma<1$, neither of the above three famous nonlinear conjugate gradient methods with the strong Wolfe line search can ensure a descent search direction even if $f$ is quadratic (see [3, 7]).

The conjugate descent (CD) method of Fletcher [14, where

$$
\beta_{k}^{C D}=\frac{\left\|g_{k}\right\|^{2}}{-d_{k-1}^{T} g_{k-1}}
$$

ensures a descent direction for general functions if the line search satisfies the strong Wolfe conditions (1.4)-(1.5) with $\sigma<1$. But the global convergence of the CD method is proved (see [8]) only for the case when the line search satisfies (1.4) and

$$
\sigma g_{k}^{T} d_{k} \leq g\left(x_{k}+\alpha_{k} d_{k}\right)^{T} d_{k} \leq 0
$$

For any positive constant $\bar{\sigma}$, an example is constructed in [8] showing that the conjugate descent method with $\alpha_{k}$ satisfying (1.4) and

$$
\sigma g_{k}^{T} d_{k} \leq g\left(x_{k}+\alpha_{k} d_{k}\right)^{T} d_{k} \leq-\bar{\sigma} g_{k}^{T} d_{k}
$$

need not converge.

Recently, Dai and Yuan [6] proposed a new conjugate gradient method, in which

$$
\beta_{k}^{D Y}=\frac{\left\|g_{k}\right\|^{2}}{d_{k-1}^{T} y_{k-1}}
$$

A remarkable property of the DY method is that it provides a descent search direction at every iteration and converges globally provided that the stepsize satisfies the Wolfe conditions, namely, (1.4) and

$$
g\left(x_{k}+\alpha_{k} d_{k}\right)^{T} d_{k} \geq \sigma g_{k}^{T} d_{k} .
$$

Some other properties of the DY method were set forth in [4, 9, 11, 12.

In [11, Dai and Yuan proposed a family of globally convergent conjugate methods, in which

$$
\beta_{k}=\frac{\left\|g_{k}\right\|^{2}}{\lambda\left\|g_{k-1}\right\|^{2}+(1-\lambda) d_{k-1}^{T} y_{k-1}},
$$


where $\lambda \in[0,1]$ is a parameter. [12] further studied the case when $\lambda \in(-\infty,+\infty)$, and proved that the family of methods using line searches that satisfy (1.4) and (1.11) converges globally if the parameters $\sigma, \bar{\sigma}$, and $\lambda$ are such that

$$
\sigma-1 \leq(\sigma+\bar{\sigma}) \lambda \leq 1 .
$$

Another marginal but interesting note on the DY method is that the formula (1.12) for $\beta_{k}$ has the same numerator as (1.6) and the same denominator as (1.8) (see [6]). In [23], Nazareth regarded the FR, PRP, HS, and DY formulae as the four leading contenders for the scalar $\beta_{k}$, and proposed a two-parameter family of conjugate gradient methods:

$$
\beta_{k}=\frac{\lambda_{k}\left\|g_{k}\right\|^{2}+\left(1-\lambda_{k}\right) g_{k}^{T} y_{k-1}}{\mu_{k}\left\|g_{k-1}\right\|^{2}+\left(1-\mu_{k}\right) d_{k-1}^{T} y_{k-1}},
$$

where $\lambda_{k}, \mu_{k} \in[0,1]$ are parameters.

In this paper, we will propose a three-parameter family of conjugate gradient methods, which includes the five nonlinear conjugate gradient methods mentioned above and the one in [22]. The three-parameter family of methods also has several other families of conjugate gradient methods and some hybrid methods as its special cases (see the next section). In Section 3, we study the descent property of the three-parameter family of methods. We prove that, if Powell's restart criterion [24] is used, the three-parameter family of methods with the strong Wolfe line search produces a descent search direction at every iteration unless the current point is a stationary point. In Section 4, some general convergence results are established for the three-parameter family of methods. Brief discussions are given in the last section.

\section{A threE-PARAMETER FAMILY OF CONJUGATE GRADIENT METHODS}

In [22], Liu and Storey presented the following formula for the scalar $\beta_{k}$ :

$$
\beta_{k}^{L S}=\frac{g_{k}^{T} y_{k-1}}{-d_{k-1}^{T} g_{k-1}} .
$$

A useful property of formulae (1.8) and (2.1) in computations is observed in 22. Namely, the next direction $d_{k+1}$ in (1.2) is independent of the length of $d_{k}$ when $\beta_{k}$ takes the form of (1.8) or (2.1).

Many authors have presented other choices for the scalar $\beta_{k}$, for example Buckley and Lenir 22], Daniel [13, Gilbert and Nocedal [16], Qi et al. 28], Shanno [29, and Touati-Ahmed and Storey [30]. Observing that the formulae (1.6)-(1.9), (1.12) and (2.1) share two numerators and three denominators, we can use combinations of these numerators and denominators to obtain the following three-parameter family:

$$
\beta_{k}=\frac{\left(1-\lambda_{k}\right)\left\|g_{k}\right\|^{2}+\lambda_{k} g_{k}^{T} y_{k-1}}{\left(1-\mu_{k}-\omega_{k}\right)\left\|g_{k-1}\right\|^{2}+\mu_{k} d_{k-1}^{T} y_{k-1}-\omega_{k} d_{k-1}^{T} g_{k-1}},
$$

where $\lambda_{k} \in[0,1], \mu_{k} \in[0,1]$ and $\omega_{k} \in\left[0,1-\mu_{k}\right]$ are parameters. Because

$$
g_{k}^{T} y_{k-1}=\left\|g_{k}\right\|^{2}-g_{k}^{T} g_{k-1}
$$

and

$$
g_{k-1}^{T} d_{k-1}=-\left\|g_{k-1}\right\|^{2}+\beta_{k-1} g_{k-1}^{T} d_{k-2},
$$


we can rewrite (2.2) as

$$
\beta_{k}=\frac{\left\|g_{k}\right\|^{2}-\lambda_{k} g_{k}^{T} g_{k-1}}{\left\|g_{k-1}\right\|^{2}+\mu_{k} g_{k}^{T} d_{k-1}-\omega_{k} \beta_{k-1} g_{k-1}^{T} d_{k-2}}
$$

If the objective function is convex quadratic and the stepsize is the exact onedimensional minimizer, the above formula for $\beta_{k}$ clearly reduces to the FR formula (1.6), since in this case we have that

$$
g_{k}^{T} d_{k-1}=0
$$

and

$$
g_{k}^{T} g_{k-1}=0 .
$$

However, for general functions, relations (2.6) and (2.7) need not hold. Therefore the methods (1.2), (1.3) and (2.5) with different values of $\lambda_{k}, \mu_{k}$ and $\omega_{k}$ form a three-parameter family of nonlinear conjugate gradient methods.

It is easy to see from (2.2) that the three-parameter family of methods includes the six already known simple and practical nonlinear conjugate gradient methods as an extreme case. If $\omega_{k} \equiv 0$, then the family reduces to the two-parameter family of conjugate gradient methods in 23$]$. Further, if $\lambda_{k} \equiv 0, \mu_{k}=\mu$ and $\omega_{k} \equiv 0$, then the family reduces to the one-parameter family in 11. Therefore the three-parameter family has the one-parameter family in [11] and the two-parameter family in [23] as its subfamilies.

In addition, the hybrid methods in [6, 16, 19] can also be regarded as special cases of the three-parameter family. For example, to combine the nice global convergence properties of the FR method and the good numerical performances of the PRP method, Hu and Storey [19] proposed a hybrid method, where

$$
\beta_{k}=\max \left\{0, \min \left\{\beta_{k}^{P R P}, \beta_{k}^{F R}\right\}\right\} .
$$

One can easily see that formula (2.8) corresponds to (2.5) with

$$
\begin{aligned}
\lambda_{k} & =\left\{\begin{array}{cl}
\frac{\left\|g_{k}\right\|^{2}}{g_{k}^{T} g_{k-1}}, & \text { if } g_{k}^{T} g_{k-1} \geq\left\|g_{k}\right\|^{2} ; \\
1, & \text { if } g_{k}^{T} g_{k-1} \in\left(0,\left\|g_{k}\right\|^{2}\right) ; \\
0, & \text { if } g_{k}^{T} g_{k-1} \leq 0,
\end{array}\right. \\
\mu_{k} & \equiv 0, \\
\omega_{k} & \equiv 0 .
\end{aligned}
$$

\section{Powell's Restart CRITERion AND DESCEnt PROPERTy}

As mentioned in the first section, if we only require that $\sigma<1$, any of the FR, PRP, and HS methods with the strong Wolfe line search may produce ascent search directions even if the objective function is quadratic. Thus, special attention must be given to the problem of how to keep the descent property of conjugate gradient methods. In this section, we will prove that, if Powell's restart criterion [24] is applied, the three-parameter family of methods with the strong Wolfe line search can guarantee the descent property of each search direction.

When dealing with Beale's three-term conjugate gradient method, Powell [24] suggested a restart with $d_{k}=-g_{k}$ if the following condition is satisfied:

$$
\left|g_{k}^{T} g_{k-1}\right| \leq \xi\left\|g_{k}\right\|^{2},
$$


where $\xi>0$ is some positive constant. As Powell 24] observed, such a restart criterion can ensure that Beale's recurrence does not converge to a non-stationary point (a strict convergence result was given in [10] for Beale's method with Powell's restart criterion), and improve the numerical behavior of Beale's method. In fact, for standard conjugate gradient methods, if the function is convex quadratic and the line search is exact, then relation (2.7) implies that no restarts would take place and finite termination could occur. Thus, the quantity $\left|g_{k}^{T} g_{k-1}\right| /\left\|g_{k}\right\|^{2}$ would indicate strong local nonquadratic behavior and hence would be indicative of a need for restarting. In the implementations of conjugate gradient methods, Powell's restart criterion has been used by many authors, for example Buckley and Lenir [2] and Khoda et al. 20.

In the following, to show the importance of Powell's restart criterion in keeping the descent property of conjugate gradient methods, we first take the HS method as an illustrative example. For this purpose, we define

$$
r_{k}=-\frac{g_{k}^{T} d_{k}}{\left\|g_{k}\right\|^{2}} .
$$

It is obvious that $d_{k}$ is a descent direction if and only if $r_{k}>0$. For the HS method (1.2), (1.3) and (1.8), direct calculations yield

$$
r_{k}=\frac{-g_{k-1}^{T} d_{k-1}}{d_{k-1}^{T} y_{k-1}}\left[1-\frac{g_{k}^{T} g_{k-1}}{\left\|g_{k}\right\|^{2}} \frac{g_{k}^{T} d_{k-1}}{g_{k-1}^{T} d_{k-1}}\right] .
$$

Suppose that $d_{k-1}$ is a descent direction and the $(k-1)$-th line search satisfies the strong Wolfe conditions (1.4)-(1.5). Then we have that $g_{k-1}^{T} d_{k-1}<0$ and $d_{k-1}^{T} y_{k-1}>0$. Furthermore, it follows from (1.5) that

$$
\left|\frac{g_{k}^{T} d_{k-1}}{g_{k-1}^{T} d_{k-1}}\right| \leq \sigma
$$

Therefore by (3.3), if Powell's restart criterion (3.2) is used, the HS method can ensure the descent property of the next direction $d_{k}$ provided that the parameters $\xi$ and $\sigma$ are such that

$$
\xi \sigma<1
$$

For the three-parameter family of conjugate gradient methods, we can prove the following general theorem.

Theorem 3.1. Consider any method in the form (1.2), (1.3) and (2.2) with $\lambda_{k} \in$ $[0,1], \mu_{k} \in[0,1]$ and $\omega_{k} \in\left[0,1-\mu_{k}\right]$, with the search condition (1.5), and with Powell's restart criterion (3.1). If the parameters $\xi$ and $\sigma$ satisfy

$$
(1+\xi) \sigma \leq \frac{1}{2}
$$

then, for all $k \geq 1$,

$$
0<r_{k}<\frac{1}{1-(1+\xi) \sigma} .
$$

Proof. Without loss of generality, assume that (3.1) holds for all $k$. We prove (3.7) by induction. Noting that $d_{1}=-g_{1}$ and hence $r_{1}=1$, we see that (3.7) is true for 
$k=1$. We now suppose that (3.7) holds for $k-1$, namely,

$$
0<r_{k-1}<\frac{1}{1-(1+\xi) \sigma} \text {. }
$$

By (1.3) and (2.5), direct calculations show that

$$
r_{k}=1-\left[1-\lambda_{k} \frac{g_{k}^{T} g_{k-1}}{\left\|g_{k}\right\|^{2}}\right] b_{k},
$$

where

$$
b_{k}=\frac{g_{k}^{T} d_{k-1}}{\left(1-\mu_{k}-\omega_{k}\right)\left\|g_{k-1}\right\|^{2}+\mu_{k} g_{k}^{T} d_{k-1}-\left(\mu_{k}+\omega_{k}\right) g_{k-1}^{T} d_{k-1}} .
$$

Using (1.5), (3.8) and the fact that $\mu_{k}, \omega_{k} \geq 0$ in (3.9), we get that

$$
\begin{aligned}
b_{k} & \leq \frac{-\sigma g_{k-1}^{T} d_{k-1}}{\left(1-\mu_{k}-\omega_{k}\right)\left\|g_{k-1}\right\|^{2}-\sigma \mu_{k} g_{k-1}^{T} d_{k-1}-\left(\mu_{k}+\omega_{k}\right) g_{k-1}^{T} d_{k-1}} \\
& =\frac{\sigma r_{k-1}}{\left(1-\mu_{k}-\omega_{k}\right)+\left[(1+\sigma) \mu_{k}+\omega_{k}\right] r_{k-1}} \\
& \leq \frac{\sigma[1-(1+\xi) \sigma]^{-1}}{\left(1-\mu_{k}-\omega_{k}\right)+\left[(1+\sigma) \mu_{k}+\omega_{k}\right][1-(1+\xi) \sigma]^{-1}} \\
& \leq \frac{\sigma}{[1-(1+\xi) \sigma]+(2+\xi) \sigma \mu_{k}+(1+\xi) \sigma \omega_{k}} \\
& \leq \frac{\sigma}{1-(1+\xi) \sigma} .
\end{aligned}
$$

Similarly, we can prove that

$$
b_{k}>-\frac{\sigma}{1-(1+\xi) \sigma} .
$$

Thus from (3.9), (3.11), (3.12), (3.1), (3.6) and the fact that $\lambda_{k} \in[0,1]$, we obtain

$$
0 \leq 1-\frac{(1+\xi) \sigma}{1-(1+\xi) \sigma}<r_{k}<1+\frac{(1+\xi) \sigma}{1-(1+\xi) \sigma}=\frac{1}{1-(1+\xi) \sigma} .
$$

Therefore (3.7) is also true for $k$. By induction, (3.7) holds for all $k \geq 1$.

In real computations, Powell 24 suggested the value of $\xi$ in (3.1) could be $\xi=0.2$. Here we should point out that condition (3.6) allows relatively large values of $\xi$ and hence is flexible, because the parameter $\sigma$ in (1.5) is generally set to a small value, normally $\sigma=0.1$.

\section{Convergence properties}

In this section, we study the global convergence properties of the three-parameter family of nonlinear conjugate gradient methods. For convenience, we assume that $g_{k} \neq 0$ for all $k$, for otherwise a stationary point has been found. We also assume that $\beta_{k} \neq 0$ for all $k$. This is because the direction in (1.3) reduces to $-g_{k}$ if $\beta_{k}=0$. Then either as the new starting point, we can take $x_{\bar{k}}$ where $\bar{k}$ is the largest index for which $\beta_{\bar{K}}=0$, or the convergence relation liminf $\left\|g_{k}\right\|=0$ holds.

The following assumption is imposed on the objective function throughout this section. 
Assumption 4.1. (i) The level set $\mathcal{L}=\left\{\S \in \Re \backslash:\left\{(\S) \leq\left\{\left(\S_{\infty}\right)\right\}\right.\right.$ is bounded.

(ii) In some neighborhood $\mathcal{N}$ of $\mathcal{L}, f$ is differentiable and its gradient $g$ is Lipschitz continuous, namely, there exists a positive constant $L$ such that

$$
\|g(x)-g(y)\| \leq L\|x-y\|, \quad \text { for all } x, y \in \mathcal{N} .
$$

The above assumption implies that there exists a positive constant $\bar{\gamma}$ such that

$$
\|g(x)\| \leq \bar{\gamma}, \quad \text { for all } x \in \mathcal{L}
$$

To give the first convergence result for the three-parameter family of methods, we need the following lemma, which can be proved similarly to Theorem 3.3 in [11.

Lemma 4.2. Suppose that $x_{1}$ is a starting point for which Assumption 4.1 holds. Consider the method (1.2)-(1.3), where $d_{k}$ is a descent direction and $\alpha_{k}$ satisfies the Wolfe conditions (1.4) and (1.13). If there exists a positive sequence $\left\{\phi_{k}\right\}$ such that

$$
\left|\beta_{k}\right| \leq \frac{\phi_{k}}{\phi_{k-1}}
$$

and

$$
\sum_{k \geq 1} \frac{\left\|g_{k}\right\|^{2}}{\phi_{k}^{2}}=+\infty,
$$

then the method converges in the sense that

$$
\liminf _{k \rightarrow \infty}\left\|g_{k}\right\|=0 \text {. }
$$

By Lemma 4.2, we can prove the following general result for the three-parameter family of nonlinear conjugate gradient methods.

Theorem 4.3. Suppose that $x_{1}$ is a starting point for which Assumption 4.1 holds. Consider any method in the form (1.2), (1.3) and (2.2) with $\lambda_{k} \in[0,1], \mu_{k} \in[0,1]$ and $\omega_{k} \in\left[0,1-\mu_{k}\right]$, with the strong Wolfe line search (1.4)-(1.5), and with Powell's restart criterion (3.1). Denote $l_{k}=\left|\beta_{k} / \beta_{k}^{F R}\right|$. If (3.6) holds, and if the parameters $\lambda_{k}, \mu_{k}$ and $\omega_{k}$ are such that the inequality

$$
\prod_{j=2}^{k} l_{j} \leq \tau \sqrt{k}
$$

holds for some constant $\tau>0$ and all $k \geq 2$, then the method converges in the sense that (4.5) holds.

Proof. Since the parameters $\xi$ and $\sigma$ satisfy (3.6), we have by Theorem 3.1 that (3.7) holds for all $k$, which implies that each $d_{k}$ is a descent direction. Define

$$
\phi_{k}=\left(\prod_{j=2}^{k} l_{j}\right)\left\|g_{k}\right\|^{2} .
$$

Then by (1.6) and the definition of $l_{k}$, we can write

$$
\left|\beta_{k}\right|=\frac{\phi_{k}}{\phi_{k-1}} .
$$

It follows from (4.2), (4.6) and 4.7) that

$$
\frac{\left\|g_{k}\right\|^{2}}{\phi_{k}^{2}} \geq \frac{1}{\tau^{2} \bar{\gamma}^{2} k},
$$


which implies the truth of (4.4). Therefore, by Lemma 4.2, (4.5) holds.

Now we discuss some special choices that satisfy condition (4.6). As mentioned in the last paragraph of Section 2, the hybrid methods in [16] and [19] can be regarded as special cases of the three-parameter family of conjugate gradient methods. Using the above theorem, we can again deduce the global convergence of the hybrid methods. For example, for the hybrid method (2.8) we have $0 \leq l_{k} \leq 1$, which indicates that (4.6) holds with $\tau=1$. Hence (4.5) holds.

Assume that $\prod_{j=2}^{k-1} l_{j} \leq \tau \sqrt{k-1}$ for some $k \geq 3$. Then at the $k$-th iteration, (4.6) must hold if

$$
l_{k} \leq \sqrt{1+\frac{1}{k-1}}
$$

Note that if $\lambda_{k}=\mu_{k}=\omega_{k}=0$, then $\beta_{k}$ reduces to $\beta_{k}^{F R}$, and hence $l_{k}=1$. Thus even for large $k$, we can see from (4.10) that there exists some interval in [0,1] for each of the parameters $\lambda_{k}, \mu_{k}$ and $\omega_{k}$ such that (4.6) holds. Though there always exists choices of $\lambda_{k}, \mu_{k}$ and $\omega_{k}$ that satisfy (4.6) (as stated above), this restriction may reduce the admissible intervals for $\lambda_{k} \in[0,1], \mu_{k} \in[0,1]$, and $\omega_{k} \in\left[0,1-\mu_{k}\right]$. Generally, for some $k$ the value of $l_{k}$ may be less than 1 , and as a result, this will allow relatively large values of the consequent $l_{k}$. For example, if the step $\left\|x_{k}-x_{k-1}\right\|$ is very small and $\lambda_{k}$ is close to 1 at some iteration far away from the solution, then $\beta_{k}$ and hence $l_{k}$ may be much smaller than 1 . Another point that should be mentioned here is that we can also enlarge the admissible intervals for $\lambda_{k}, \mu_{k}$ and $\omega_{k}$ by setting $\tau$ in (4.6) equal to a large value. Inequality (4.6) suggests that one possibility is to choose $\lambda_{k}, \mu_{k}$ and $\omega_{k}$ such that the absolute value of the right hand side of (2.2) is as small as possible.

By Lemma 4.2 we can also prove the following convergence result.

Theorem 4.4. Suppose that $x_{1}$ is a starting point for which Assumption 4.1 holds. Consider any method in the form (1.2), (1.3) and (2.2) with $\lambda_{k} \in[0,1], \mu_{k} \in[0,1]$ and $\omega_{k} \in\left[0,1-\mu_{k}\right]$, with the strong Wolfe line search (1.4) -(1.5), and with Powell's restart criterion (3.1). If (3.6) holds, and if the parameters $\lambda_{k}, \mu_{k}$ and $\omega_{k}$ are such that

$$
0 \leq \lambda_{k} g_{k}^{T} g_{k-1} \leq\left\|g_{k}\right\|^{2}
$$

and

$$
\mu_{k} g_{k}^{T} d_{k-1}-\omega_{k} \beta_{k-1} g_{k-1}^{T} d_{k-2} \geq-\lambda_{k-1} g_{k-1}^{T} g_{k-2}
$$

for all $k \geq 2$, then the method converges in the sense that (4.5) holds.

Proof. Since the parameters $\xi$ and $\sigma$ satisfy (3.6), we have by Theorem 3.1 that (3.7) holds for all $k$, which implies that each $d_{k}$ is a descent direction. From (4.11), (4.12) with $k$ replaced by $k+1$, and the equivalent formula (2.5) of $\beta_{k}$, we see that (4.3) holds with

$$
\phi_{k}=\left(1-\mu_{k+1}-\omega_{k+1}\right)\left\|g_{k}\right\|^{2}+\mu_{k+1} d_{k}^{T} y_{k}-\omega_{k+1} d_{k}^{T} g_{k} .
$$


Using (1.5) and (3.7) in (4.13), we can prove that

$$
\begin{aligned}
\phi_{k} & \leq\left[\left(1-\mu_{k+1}-\omega_{k+1}\right)+(1+\sigma) \mu_{k+1} r_{k}+\omega_{k+1} r_{k}\right]\left\|g_{k}\right\|^{2} \\
& \leq\left[1+(2+\sigma)(1-(1+\xi) \sigma)^{-1}\right]\left\|g_{k}\right\|^{2} \\
& =\frac{3-\xi \sigma}{1-(1+\xi) \sigma}\left\|g_{k}\right\|^{2}
\end{aligned}
$$

for any $\mu_{k+1} \in[0,1]$ and $\omega_{k+1} \in\left[0,1-\mu_{k+1}\right]$. The above relation and (4.2) imply that

$$
\sum_{k \geq 1} \frac{\left\|g_{k}\right\|^{2}}{\phi_{k}^{2}}=+\infty
$$

Thus, by Lemma 4.2, (4.5) holds.

If $\xi \leq 1$, the second inequality in (4.11) clearly holds, since by (3.1) and the fact that $\lambda_{k} \in[0,1]$ we have

$$
\lambda_{k} g_{k}^{T} g_{k-1} \leq\left|g_{k}^{T} g_{k-1}\right| \leq \xi\left\|g_{k}\right\|^{2} \leq\left\|g_{k}\right\|^{2} .
$$

It is easy to see that the FR method $\left(\lambda_{k}=\mu_{k}=\omega_{k}=0\right)$ satisfies the two conditions (4.11) and (4.12). However, for other methods it is not clear whether these conditions hold, as these conditions depend on the sequences of points generated by the methods. For example, in the extreme case $g_{k}^{T} g_{k-1}<0, g_{k}^{T} d_{k-1}<0$ and $\beta_{k-1} g_{k-1}^{T} d_{k-2}>0$, we need to choose $\lambda_{k}=\mu_{k+1}=\omega_{k+1}=0$. If $g_{k}^{T} g_{k-1}>0$, there always exist admissible intervals for $\lambda_{k}, \mu_{k+1}$ and $\omega_{k+1}$.

The following lemma is drawn from Gilbert and Nocedal [16].

Lemma 4.5. Suppose that $x_{1}$ is a starting point for which Assumption 4.1 holds. Consider any method in the form (1.2)-(1.3) with the following three properties:

(i) $\beta_{k} \geq 0$

(ii) The Wolfe conditions (1.4) and (1.13) and the sufficient descent condition $g_{k}^{T} d_{k} \leq-c\left\|g_{k}\right\|^{2}$ hold for all $k$ and some positive constant $c$.

(iii) Property $(*)$ holds; namely, there exist constants $b>1$ and $\lambda>0$ such that $\left|\beta_{k}\right| \leq b$ for all $k$, and if $\left\|x_{k}-x_{k-1}\right\| \leq \lambda$, then $\left|\beta_{k}\right| \leq(2 b)^{-1}$.

Then the method converges in the sense that (4.5) holds.

By Lemma 4.5, we can prove the following general result for our three-parameter family of nonlinear conjugate gradient methods.

Theorem 4.6. Suppose that $x_{1}$ is a starting point for which Assumption [4.1 holds. Consider any method in the form (1.2), (1.3) and (2.2), where $\lambda_{k} \in[0,1], \mu_{k} \in[0,1]$ and $\omega_{k} \in\left[0,1-\mu_{k}\right]$, where the stepsize satisfies the strong Wolfe conditions (1.4)(1.5), and where the restart criterion

$$
-\xi\left\|g_{k}\right\|^{2} \leq g_{k}^{T} g_{k-1} \leq\left\|g_{k}\right\|^{2}
$$

is used. If the parameters are such that

$$
(1+\xi) \sigma<\frac{1}{2}
$$

and

$$
\lambda_{k} \geq 1-c_{1}\left\|x_{k}-x_{k-1}\right\|,
$$

where $c_{1}>0$ is constant, then the method converges in the sense that (4.5) holds. 
Proof. We proceed by contradiction. Assume that

$$
\liminf _{k \rightarrow \infty}\left\|g_{k}\right\| \neq 0
$$

Then there exists a positive constant $\gamma$ such that

$$
\left\|g_{k}\right\| \geq \gamma, \quad \text { for all } k \geq 1 .
$$

Using (4.17) and (4.18), we can see from (3.13) in the proof of Theorem 3.1 that, for all $k \geq 1$,

$$
r_{k} \geq \frac{1-2(1+\xi) \sigma}{1-(1+\xi) \sigma} \triangleq c_{2}
$$

where $r_{k}$ is given in (3.2). Thus the sufficient descent condition holds. From (1.5), (3.7), (4.1), (4.2), (4.21) and the fact that $\lambda_{k} \leq 1$, we can show that

$$
\begin{aligned}
\left|\beta_{k}\right| & \leq \frac{\left(c_{1}\left\|g_{k}\right\|^{2}+L\left\|g_{k}\right\|\right)\left\|x_{k}-x_{k-1}\right\|}{\left\|g_{k-1}\right\|^{2}\left[\left(1-\mu_{k}-\omega_{k}\right)+\mu_{k}(1-\sigma) c_{2}+\omega_{k} c_{2}\right]} \\
& \leq \frac{\left(c_{1}\left\|g_{k}\right\|^{2}+L\left\|g_{k}\right\|\right)\left\|x_{k}-x_{k-1}\right\|}{c_{2}\left\|g_{k-1}\right\|^{2}} \\
& \leq c_{3}\left\|x_{k}-x_{k-1}\right\|,
\end{aligned}
$$

where

$$
c_{3}=\frac{c_{1} \bar{\gamma}^{2}+L \bar{\gamma}}{c_{2} \gamma^{2}}
$$

Since Assumption 4.1 implies that there exists a positive constant $\rho$ such that

$$
\|x\| \leq \rho, \quad \text { for all } x \in \mathcal{L},
$$

for $b=2 c_{3} \rho$ amd $\lambda=\left(4 c_{3}^{2} \rho\right)^{-1}$, we have from (4.23) and (4.25) that

$$
\left|\beta_{k}\right| \leq b
$$

and, if $\left\|x_{k}-x_{k-1}\right\| \leq \lambda$,

$$
\left|\beta_{k}\right| \leq(2 b)^{-1} \text {. }
$$

Thus Property $(*)$ holds. In addition, (3.7) and (4.17) imply that $\beta_{k} \geq 0$. Therefore the conditions of Lemma 4.5 are all satisfied, and hence (4.5) holds.

Theorems 4.3 4.4 and 4.6 provide some general convergence results for the threeparameter family of nonlinear conjugate gradient methods. If the parameters in (2.2) are specifically chosen, then better global convergence results can be expected. We will end this section with such an illustrative example.

Such an example stems from the following fact. For any method of the form (1.2) - (1.3) with $d_{k}$ satisfying $g_{k}^{T} d_{k}<0$ and with the strong Wolfe line search (1.4)(1.5), Corollary 2.4 in [5] tells us that the method converges globally provided that the norm of $d_{k}$ does not increase faster than linearly. Specifically, the method gives (4.5) if the following condition holds:

$$
\sum_{k \geq 1} \frac{1}{\left\|d_{k}\right\|^{2}}=+\infty .
$$


Therefore, in the three-parameter family of nonlinear conjugate gradient methods, to shorten the length of $d_{k}$, it is reasonable to choose the parameters so that $\left|\beta_{k}\right|$ reaches its smallest value. Namely, let

$$
\left(\bar{\lambda}_{k}, \bar{\mu}_{k}, \bar{\omega}_{k}\right)=\operatorname{argmin}_{\lambda_{k}, \mu_{k} \in[0,1], \omega_{k} \in\left[0,1-\mu_{k}\right]}\left|\beta_{k}\right|,
$$

where $\beta_{k}$ is defined in (2.2). In this case, we can obtain the following global convergence result, in which the line search only needs to satisfy the Wolfe conditions.

Theorem 4.7. Suppose that $x_{1}$ is a starting point for which Assumption 4.1 holds. Consider the method of the form (1.2), (1.3) and

$$
\bar{\beta}_{k}=\frac{\left(1-\bar{\lambda}_{k}\right)\left\|g_{k}\right\|^{2}+\bar{\lambda}_{k} g_{k}^{T} y_{k-1}}{\left(1-\bar{\mu}_{k}-\bar{\omega}_{k}\right)\left\|g_{k-1}\right\|^{2}+\bar{\mu}_{k} d_{k-1}^{T} y_{k-1}-\bar{\omega}_{k} d_{k-1}^{T} g_{k-1}},
$$

where $\bar{\lambda}_{k}, \bar{\mu}_{k}, \bar{\omega}_{k}$ are given in (4.29). If the stepsize satisfies the Wolfe conditions (1.4) and (1.13), then each $d_{k}$ generated by the method is a descent direction. Further, the method converges in the sense that (4.5) holds.

Proof. From the choices of $\bar{\lambda}_{k}, \bar{\mu}_{k}$ and $\bar{\omega}_{k}$, it is easy to see that

$$
0 \leq \bar{\beta}_{k} \leq \beta_{k}^{D Y},
$$

where $\beta_{k}^{D Y}$ is given in (1.12). Thus the statements follow by Theorem 3.1 in $[6]$.

\section{Conclusions And Discussions}

In this paper, we have proposed a three-parameter family of nonlinear conjugate gradient methods, and studied the global convergence of these methods. The threeparameter family not only includes the six already known simple and practical conjugate gradient methods, but has some other families of conjugate gradient methods as subfamilies. The three-parameter family also includes some hybrid methods as special cases.

With Powell's restart criterion, we proved that the three-parameter family can ensure a descent search direction at every iteration. Then, under suitable conditions, we established some general convergence results, namely, Theorems 4.3, 4.4 and 4.6, for the three-parameter family of nonlinear conjugate gradient methods. If the parameters are specifically chosen, better global convergence results could be achieved.

It has been pointed out that condition (3.6) is not strict, because the parameter $\sigma$ in (1.5) is generally chosen to be relatively small, which implies that $\xi$ in (3.1) could be relatively large. However, it still remains to explore how to find new and efficient conjugate gradient methods among the three-parameter family. Specifically, it would be interesting to find the practical performance of the method (4.29).

\section{ACKNOWLEDGMENTS}

The authors would like to thank an anonymous referee for comments and suggestions which improved this paper. 


\section{REFERENCES}

[1] M. Al-Baali, Descent property and global convergence of the Fletcher-Reeves method with inexact line search, IMA J. Numer. Anal. 5 (1985), pp. 121-124. MR 86d:49043

[2] A. Buckley and A. Lenir, QN-like variable storage conjugate gradients, Math. Prog. 27 (1983), 155-175. MR 85a:49043

[3] Y. H. Dai, Analyses of nonlinear conjugate gradient method, Ph.D. thesis, Institute of Computational Mathematics and Scientific/Engineering Computing, Chinese Academy of Sciences, 1997.

[4] Y. H. Dai, Some new properties of a nonlinear conjugate gradient method, Research report ICM-98-010, Institute of Computational Mathematics and Scientific/Engineering Computing, Chinese Academy of Sciences, 1998.

[5] Y. H. Dai, J. Y. Han, G. H. Liu, D. F. Sun, H. X. Yin, and Y. Yuan, Convergence properties of nonlinear conjugate gradient methods, Research report ICM-98-024, Institute of Computational Mathematics and Scientific/Engineering Computing, Chinese Academy of Sciences, 1998 (accepted by SIAM J. Optimization).

[6] Y. H. Dai and Y. Yuan, A Nonlinear Conjugate Gradient Method with Nice Global Convergence Properties, Research report ICM-95-038, Institute of Computational Mathematics and Scientific/Engineering Computing, Chinese Academy of Sciences, 1995 (accepted by SIAM J. Optimization).

[7] Y. H. Dai and Y. Yuan, Convergence properties of the Fletcher-Reeves method, IMA J. Numer. Anal. Vol. 16 No. 2 (1996), 155-164. MR 97i:65099

[8] Y. H. Dai and Y. Yuan, Convergence properties of the conjugate descent method, Mathematical Advances Vol. 25 No. 6 (1996), 552-562. CMP 97:13

[9] Y. H. Dai and Y. Yuan, Some properties of a new conjugate gradient method, in: Y. Yuan ed., Advances in Nonlinear Programming (Kluwer, Boston, 1998), pp. 251-262. MR 99b:90004

[10] Y. H. Dai and Y. Yuan, Convergence properties of Beale-Powell restart method, Sciences in China (series A), Vol. 28, No. 5, pp. 424-432.

[11] Y. H. Dai and Y. Yuan, A class of globally convergent conjugate gradient methods, Research report ICM-98-030, Institute of Computational Mathematics and Scientific/Engineering Computing, Chinese Academy of Sciences, 1998.

[12] Y. H. Dai and Y. Yuan, Extension of a class of conjugate gradient methods, Research report ICM-98-049, Institute of Computational Mathematics and Scientific/Engineering Computing, Chinese Academy of Sciences, 1998.

[13] J. W. Daniel, The conjugate gradient method for linear and nonlinear operator equations, SIAM J. Numer. Anal., 4 (1967), 10-26. MR 36:1076

[14] R. Fletcher, Practical Methods of Optimization vol. 1: Unconstrained optimization, John Wiley \& Sons (New York), 1987. MR 83i:65055a

[15] R. Fletcher and C. Reeves, Function minimization by conjugate gradients, Comput. J. 7 (1964), pp. 149-154. MR 32:4827

[16] J. C. Gilbert and J. Nocedal, Global convergence properties of conjugate gradient methods for optimization, SIAM. J. Optimization. Vol. 2 No. 1 (1992), pp. 21-42. MR 92k:90089

[17] L. Grippo and S. Lucidi, A globally convergent version of the Polak-Ribière conjugate gradient method, Math. Prog. 78 (1997), pp. 375-391. MR 98k:90080

[18] M. R. Hestenes and E. L. Stiefel, Methods of conjugate gradients for solving linear systems, J. Res. Nat. Bur. Standards Sect. 5, 49 (1952), 409-436. MR 15:651a

[19] Y. F. Hu and C. Storey, Global convergence result for conjugate gradient methods, J. Optim. Theory Appl. Vol. 71 No. 2 (1991) 399-405. MR 92i:90095

[20] K. M. Khoda, Y. Liu, and C. Storey, Generalized Polak-Ribire Algorithm, Journal of Optimization Theory and Applications, Vol. 75, No. 2 (1992), 345-354. CMP 93:04

[21] G. H. Liu, J. Y. Han, and H. X. Yin, Global convergence of the Fletcher-Reeves algorithm with an inexact line search, Appl. Math. J. Chinese Univ. Ser. B, 10 (1995), 75-82. MR 96b:90086

[22] Y. Liu and C. Storey, Efficient Generalized Conjugate Gradient Algorithms, Part 1: Theory, Journal of Optimization Theory and Applications, Vol. 69 (1991), 129-137. MR 92e:90077

[23] L. Nazareth, Conjugate-gradient methods, to appear in: Encyclopedia of Optimization (C. Floudas and P. Pardalos, eds.), Kluwer Academic Publishers, Boston, USA and Dordrecht, The Netherlands (1999). 
[24] M. J. D. Powell, Restart procedures of the conjugate gradient method, Math. Program. 2 (1977), pp. 241-254. MR 57:18099

[25] M. J. D. Powell, Nonconvex minimization calculations and the conjugate gradient method, in: Lecture Notes in Mathematics vol. 1066, Springer-Verlag (Berlin) (1984), pp. 122-141. MR 85g:49035

[26] E. Polak and G. Ribière, Note sur la convergence de directions conjugées, Rev. Francaise Informat Recherche Operationelle, 3e Année 16 (1969), pp. 35-43. MR 40:8232

[27] B. T. Polyak, The conjugate gradient method in extreme problems, USSR Comp. Math. and Math. Phys. 9 (1969), pp. 94-112. MR 41:2899

[28] H. D. Qi, J. Y. Han and G. H. Liu, A modified Hestenes-Stiefel conjugate gradient algorithm, Chinese Annals of Mathematics 17A : 3 (1996), 277-284. (in Chinese) CMP 97:07

[29] D. F. Shanno, Conjugate gradient methods with inexact searches, Math. Oper. Res. 3 (1978), 244-256. MR 80d:65082

[30] D. Touati-Ahmed and C. Storey, Efficient hybrid conjugate gradient techniques, J. Optimization Theory Appl. 64 (1990) 379-397. MR 91c:65046

[31] C. Y. Wang and Y. Z. Zhang, Global convergence properties of s-related conjugate gradient methods, Report, Qufu Normal University, 1996.

State Key Laboratory of Scientific and Engineering Computing, Institute of Computational Mathematics and Scientific/Engineering Computing, Academy of Mathematics and Systems Sciences, Chinese Academy of Sciences, P. O. Box 2719, Beijing 100080, CHINA

E-mail address: dyh@lsec.cc.ac.cn

State Key Laboratory of Scientific and Engineering Computing, Institute of Computational Mathematics and Scientific/Engineering Computing, Academy of Mathematics and Systems Sciences, Chinese Academy of Sciences, P. O. Box 2719, Beijing 100080, CHINA

E-mail address: yyx@lsec.cc.ac.cn 